\title{
Nanofiller Dispersion in Polymer Dielectrics
}

\author{
Daniel Tan, Yang Cao, Enis Tuncer, Patricia Irwin \\ Dielectrics \& Electrophysics Lab, GE Global Research Center, Niskayuna, Schenectady, USA. \\ Email: tan@ge.com, tuncer@ge.com
}

Received January $9^{\text {th }}, 2013$; revised February $8^{\text {th }}, 2013$; accepted March $5^{\text {th }}, 2013$

Copyright (C) 2013 Daniel Tan et al. This is an open access article distributed under the Creative Commons Attribution License, which permits unrestricted use, distribution, and reproduction in any medium, provided the original work is properly cited.

\begin{abstract}
Nanodielectric composites have been developed in recent years attempting to improve the dielectric properties such as dielectric constant, dielectric strength and voltage endurance. Among various investigations, nanoparticle dispersion was particularly emphasized in this work. General Electric Global Research Center in Niskayuna NY USA has investigated various nanoparticles, nanocomposites and nanocomposite synthesis methods intending to understand particle dispersion and their impact on the nanocomposite dielectric properties. The breakdown strength and microstructures of the nanocomposites containing different particles were studied for projects related to capacitor and electrical insulation technologies. The nanocomposite synthesis methods either employed commerical nanoparticles or utilized nanoparticles that were self-assembled (in-situ precipitation) in a matrix. Our investigations have shown that nanocomposites prepared with solution chemistry were more favorable for producing uniform dispersion of nanoparticles. Structural information of nanocomposites was studied with transmission electron microscopy and the interection between particles and matrix polymers were tentatively probed using dielectric spectroscopy. In these new class of materials high energy densities on the order of $15 \mathrm{~J} / \mathrm{cc}$ were achievable in nanocomposites.
\end{abstract}

Keywords: Nanoparticles; Polymer; Dielectrics; Filler Dispersion; Dielectric Properties

\section{Introduction}

The rapid development of new advanced and renewable energy technologies as well as defence applications demand high energy density, high power density and high temperature components [1]. Among these components are advanced capacitors that greatly require the advanced dielectric materials and film processing techniques. It has been well recognized that every new generation of capacitors have been primarily credited to the innovation and engineering of new dielectric materials. The process cycle from dielectric materials (development and chemistry) all the way to capacitor fabrication (metallization, wounding and contact spray) is shown in Figure 1.

Conventional dielectric material development followed the paths of organic polymers, inorganic ceramics, and thin films separately, due to type of capacitors manufactured, polymeric film and multi-layer ceramic capacitors. Polymer dielectrics show very high dielectric strength $(>300 \mathrm{kV} / \mathrm{mm})$, lower dielectric losses $(<0.01)$, and adequate mechanical flexibility in processing, which is important in thin film capacitors. However, they have low relative dielectric permittivity or constant $(<4)$ and low operating temperatures $\left(<200^{\circ} \mathrm{C}\right)$ compared to ce- ramic materials. Ceramic dielectrics tend to have very high dielectric permittivity $(>100)$ but relatively low dielectric strength $(<50 \mathrm{kV} / \mathrm{mm})$ and/or may be piezoelectric associated with the structure of (ferroelectric type) ceramics. The low dielectric strength in ceramics is caused by the presence of grain boundaries, intrinsic porosity, impurities, surface defects, and chemical deterioration. Thin film dielectrics are usually in nanometer to submicron in thickness with very high breakdown strength, and they require a supporting substrate. But, they are primarily useful for low voltage and microelectronics application, which are outside the scope of our current work. Increasing the dielectric breakdown strength and ensuring scalable, and reliable films are one of the challenges and goals in modern day dielectrics research in capacitor and electrical insulation applications.

Nanodielectric composites belong to a new type of materials, engineered for improved specific functionality, such as better performance in electrical insulation. Certain ceramic materials can be selected in powder form to be blended with polymers to provide synergy between the high breakdown strength polymer and high permittivity ceramic materials as shown in Figure 2. A number of research areas have been being actively pursued in order 


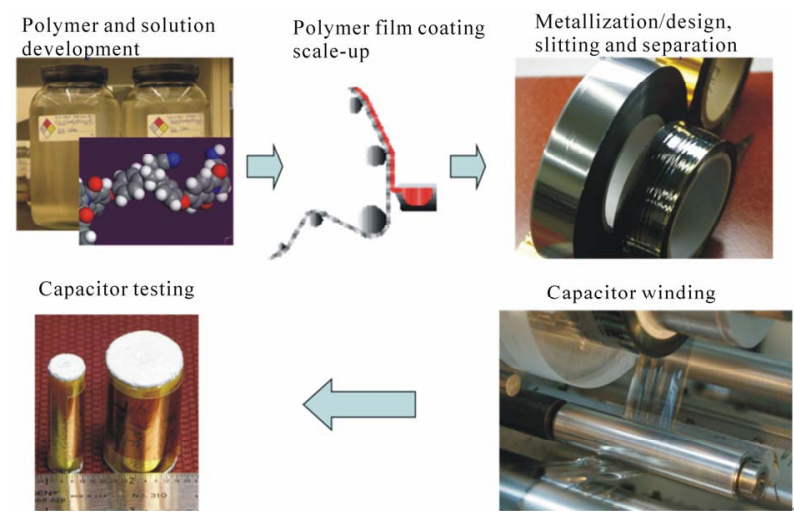

Figure 1. A sketch of capacitor manufacturing from materials to produced capacitors. Evolution of capacitor technology has a close relationship with materials development.

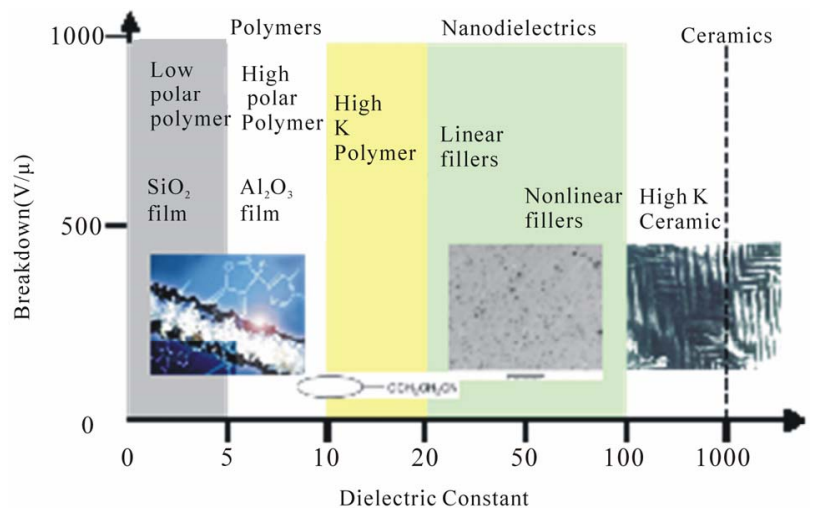

Figure 2. Relationship between breakdown strength and dielectric permittivity of various dielectric materials.

to fully explore the advantages of the functional composites for energy storage applications.

Nano-sized inorganic particles have received great attention due to their advantages in processing uniform composites and for their potential in voltage stress improvement in high voltage technology as opposed to microsized particles [2-4]. Notice that nanoparticles result in significant particle agglomeration that needs to be resolved during nanocomposite production, high interface fraction, and porosity in polymer matrices, if no special attention is given to mixing method. Porosity could result in both Maxwell-Wagner polarization at low frequencies and lower dielectric permittivities. Solving these issues will result in better nanocomposites for dielectric component and insulation applications. The current paper review the recent progresses in our labs in nanodielectric composite investigations with focus on the effect of nanofillers and its nature, size, distribution and particlepolymer interface. The particle dispersion and its importance in nanodielectric engineering is discussed. Influence of different nanocomposite synthesis methods and the resulting nanostructural features as well as the dielectric properties of selected composites are presented. The majority of the nanocomposites were prepared in wet solution method assisted with a sonication for filler dispersion. The particles were dispersed in a solvent and later introduced into a matrix polymer. For characterizations of electrical properties of nanocomposites several to tens of micrometer thick films were cast using either a solvent cast or spin coating methods. DC breakdown tests were conducted following ASTM D149 (method A) using a ball-plane electrode configuration. Structural characterization of filler shape and dispersion were studied using a transmission electron microscopy technique.

\section{Need for High Energy Density Dielectric Films}

Various government agencies have been actively solicitating dielectric materials for energy storage that can offer a high energy density of $>20 \mathrm{~J} / \mathrm{cm}^{3}$ with high operating temperature capability (as high as $200^{\circ} \mathrm{C}$ ). Examples of applications are pulsed power and power conditioning applications for electrical grid. As a response, different methods and material approaches were studied to achieve the requirements $[5,6]$. For example, a homogeneous dispersion of ferroelectric $\mathrm{BaTiO}_{3}$ particles were prepared in polypropylene with an in-situ polymerization process [3].

Although the permittivity was increased by almost $3 x$ over polypropylene, the breakdown strength of the composite was degraded. Dielectric permittivity and energy density are related via design breakdown strength of the dielectric as shown in Figure 3. Note that the dielectric loss tangent in the selected material should be low enough (lower than 1\%) over a broad frequency range. The relationship in Figure 3(a) indicates that development of better dielectric materials would be appreciated for transformational energy storage devices.

As an example, for a dielectric film to meet an energy density higher than $20 \mathrm{~J} / \mathrm{cm}^{3}$, the dielectric material needs to satisfy the conditions in Figure 3(b), one candidate may have a dielectric permittivity of $10-20$ and a high breakdown strength of $>600 \mathrm{kV} / \mathrm{mm}$. The lines in Figure 3(b) illustrate different energies densities and requirements on the dielectric properties of the candidates. If a composite material with a permittivity of higher than 60 is considered, much lower breakdown strength $(<300 \mathrm{kV} / \mathrm{mm})$ is required. Polypropylene films with relative permittivity 2.2 and design strength 800 $\mathrm{kV} / \mathrm{mm}$ result in $5.6 \mathrm{~J} / \mathrm{cc}$, indicated with red symbol in Figure 3(b). Other materials and composites are also labeled in Figure 3(b).

One of the approaches to achieve high energy densisty has been the novel dielectric materials designed using nanoengineering and nanotechnology [4,5]. These high performance nanodielectric composites have become one of the strategic building blocks in material technology in the last decade. 


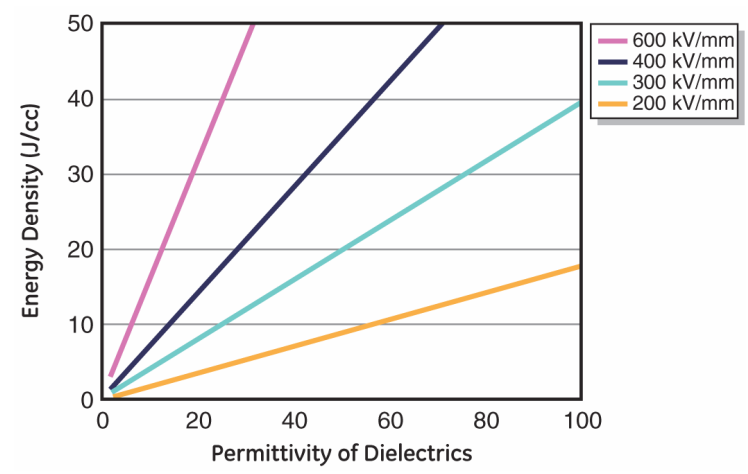

(a)

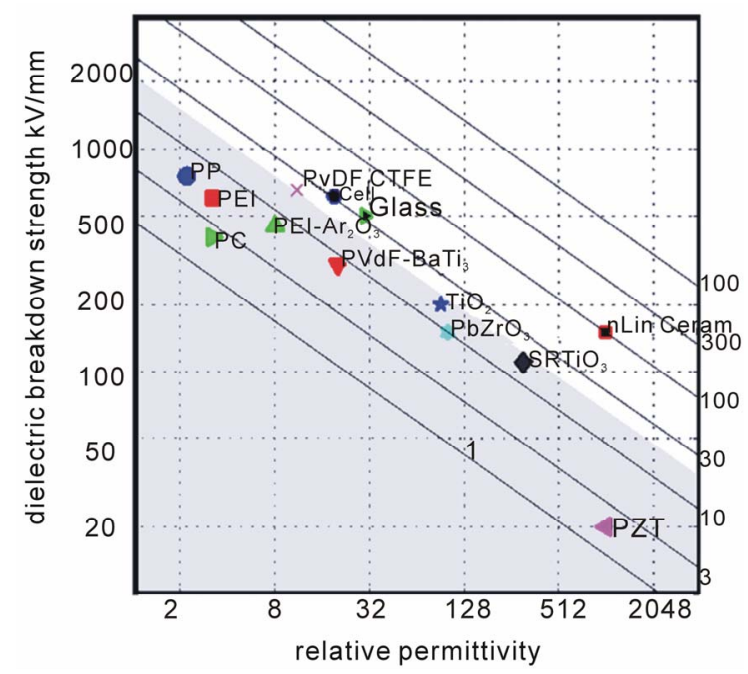

(b)

Figure 3. Energy density as a function of relative permittivity for different dielectric breakdown values. To reach the targeted energy density of $>\mathbf{2 0} \mathrm{J} / \mathrm{cm}^{3}$ for film material, high permittivity film or higher breakdown strength are needed. The figure on the right illustrates the equi-energy lines for $3, \ldots, 1000 \mathrm{~J} / \mathrm{cc}$, together with the current-state-of-the-art materials.

For this purpose, a number of research labs around the world have studied nanodielectrics to increase the dielectric permittivity of a polymers [6-9]. Even with properly selected inorganic fillers, a great succeess has not been achieved without retaining the breakdown strength and low dielectric loss for a broad frequency range. The large losses caused by mainly large mismatch in electric properties of constituents, relative permittivity and electrical conductivity, and interface between inorganic filler and polymeric matrix. In fact, simply filling a polymer matrix with high permittivity particles was found to yield a slight increase in composite effective permittivity. The shape of filler particles and their concentrations as well as their distribution are important factors to be considered besides the dielectric properties of the filler [10].

For example, an antiferroelectric type of ceramic material made of lead zirconate composition is able to ex- hibit a significantly high dielectric permittivity peak after sintering the particles at about $950^{\circ} \mathrm{C}$ or $1120^{\circ} \mathrm{C}$, respectively (Figure 4). The relative permittivity of the sintered ceramic is up to several hundred at room temperature as shown in Figure 4. After blending $10 \mathrm{wt} \%$ the powder into polyetherimide (PEI) films, the composite relative permittivity was dramatically lower, about 15 , compared to the relative permittivity of the powder as shown by the inset in Figure 4. Studies performed at General Electric Global Research Center (GE GRC) have found that high relative permittivity polymers should better be utilized in composites. However, a significant amount of effort is still required to develop materials to compete with conventional device dielectrics.

\section{Nanofiller Dispersion in Polymers}

One of the common challenges in blending hard (inorganic fillers) and soft (polymers) materials is the dispersion of filler particles. Usually, this has a lot to do with the filler surface energy. For example, the $\mathrm{SiO}_{2}$ particles were often found agglomerated in polymer matrices [11]. Mixing assisted with high energy shear force generate better results via creating enough energy to the separate agglomeration of particles [10]. A high energy sonication process has been found to be an effective method to improve the particle distribution [12-14]. Figure 5(a) shows the TEM image of $45 \mathrm{~nm} \mathrm{Al}_{2} \mathrm{O}_{3}$ particles of $5 \mathrm{vol} \%$ blended in a PEI film. Quite uniform distribution and less degree of agglomeration were obtained; observe that the particle size distribution is wide. However this method is still insufficient to disperse finer particles such as $11 \mathrm{~nm}$ $\mathrm{Al}_{2} \mathrm{O}_{3}$ as shown in Figure 5(b). The figure shows the importance of force or energy needed to separate small nanoparticles. The force is related to the particle-particle interaction and the viscosity of the medium that they were dispersed in. Utilizing surfactants or caping agents help to improve dispersion via lowering surface energy requirements.

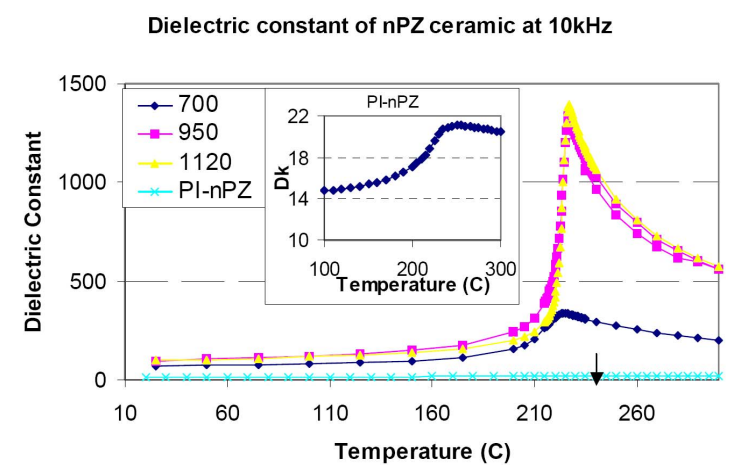

Figure 4. The temperature dependence of the relative dielectric permittivity of lead zirconate ceramics for different process conditions, and nanoparticle PZ filled polyetherimide polymer. 


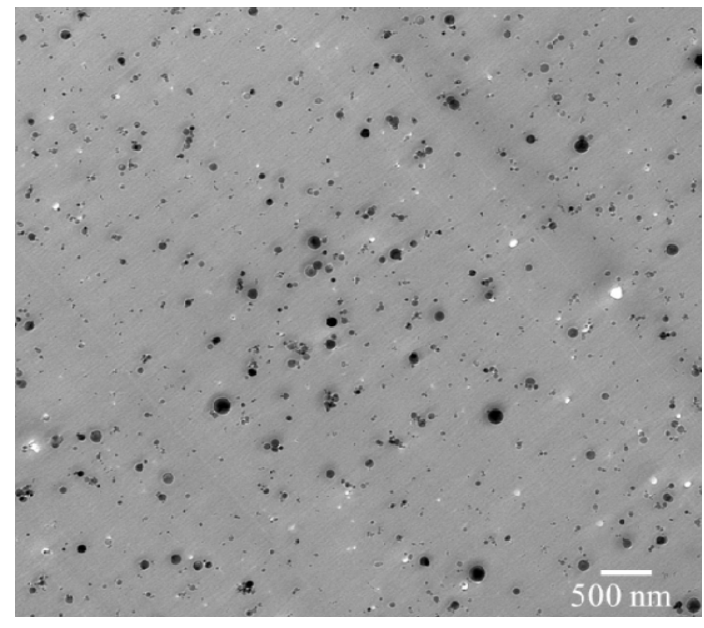

(a)

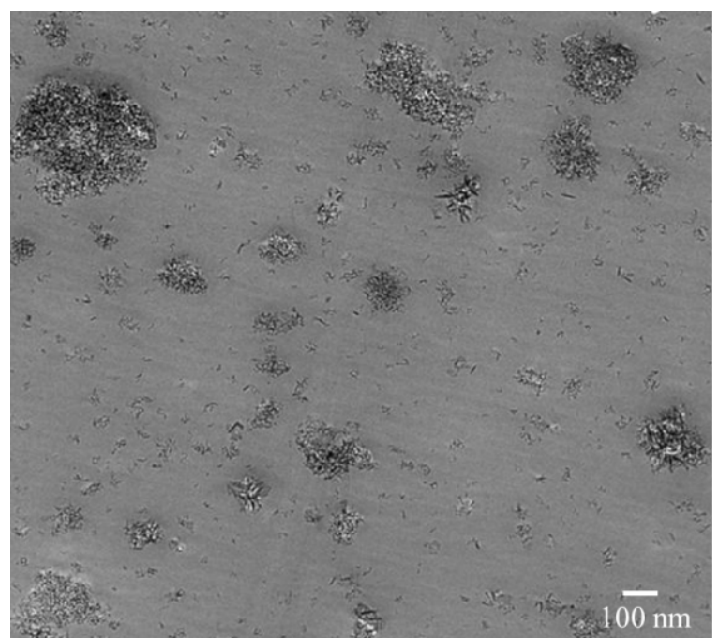

(b)

Figure 5. Transmission electron microscope images of dry nanoparticles in Ultem ${ }^{\mathrm{TM}}$. (a) $45 \mathrm{~nm} \mathrm{Al}_{2} \mathrm{O}_{3}$ and (b) $11 \mathrm{~nm}$ $\mathrm{Al}_{2} \mathrm{O}_{3}$. Observe that $\mathrm{Al}_{2} \mathrm{O}_{3}$ particles are clustered and the mixing method could not break the particle agglemorations.

It has been shown previously that one can achieve uniform dispersion of particles when nanoparticles were synthesized using solution chemisty-particle precipitation method [12]. GE GRC found that better particle dispersions could be achievable when the nanoparticles were synthesized in-house and preserved in the solvent (i.e., wet particles). The images of $\mathrm{Sb}_{2} \mathrm{O}_{3}$ and $\mathrm{SiO}_{2}$ particles of $5 \mathrm{vol} \%$ that were $<20 \mathrm{~nm}$, and dispersed in PEI polymer were shown in Figures 6(a) and (b). The particles are randomly distributed in the microscopic scale. Nevertheless, certain agglomeration of fine structure still remains due to lack of surfactant additives.

Much better particle dispersions were demonstrated by a small number of research labs utilizing in-situ polymerization process or self-assembling within a polymer matrix [15]. For in-situ polymerization process, the nanoparticles were first blended with the host monomer

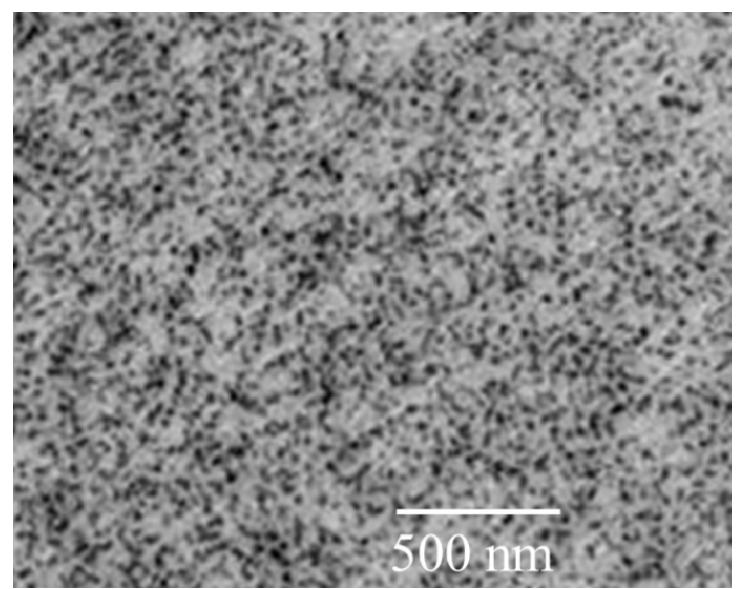

(a)

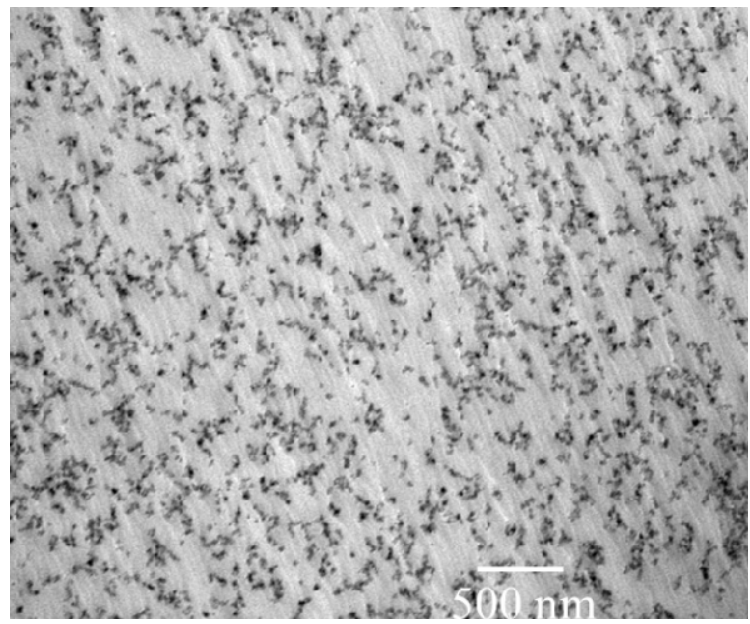

(b)

Figure 6. Transmission electron microscope images of wet $\mathrm{Sb}_{2} \mathrm{O}_{3}$ and $\mathrm{SiO}_{2}$ nanoparticles (5 vol\%) in Ultem ${ }^{\mathrm{TM}}$. The particles are better dispersed in the matrix compared to dry-powder mixed nanocomposites.

solution followed by a polymerization process. Figure 7(a) clearly shows the individual $\mathrm{SiO}_{2}$ nanoparticle distributed randomly in the host polymer, where no particle agglomeration was observed [16]. The good dispersion and adhesion of modified $\mathrm{BaTiO}_{3}$ in a polyamic matrix was also exhibited as shown in Figure 7(b) [17]. This method is effective to avoid particle agglomeration, however, the processing requires good control. This approach has not been widely adopted for nanodielectric composite processing in our labs.

Another method is in-situ self-assembling of nanoparticles in polymeric matrix. The in-situ nanoparticle precipitation method for nanocomposites has been developed for cryogenic dielectric applications by Tuncer et al. $[18,19]$. In this method, a metal salt precursor is dissolved and blended into a dissolved polymer solution. By controlling the acidity and temperature, the precursor synthesizes metal-oxide nanoparticles via chemical reac- 


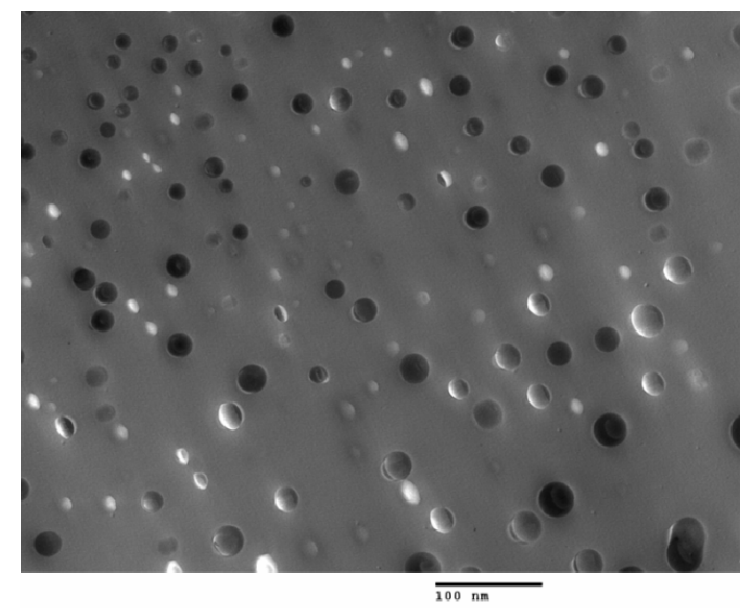

(a)

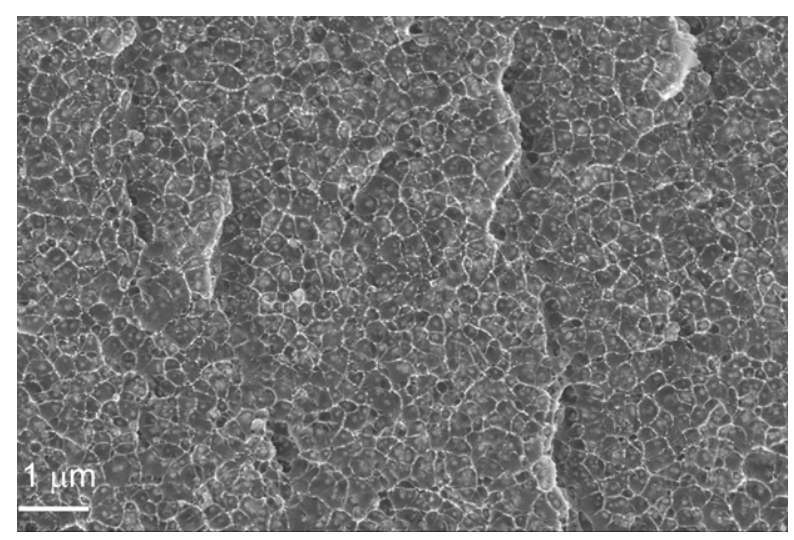

(b)

Figure 7. TEM images of nanoparticles from in-situ polermization of (a) $5 \mathrm{vol} \% \mathrm{SiO}_{2}$ in polyamicacid, and (b) 15 vol\% $40 \mathrm{~nm} \mathrm{BaTiO}_{3}$ in a polyimid containing 2,2-Bis[4-(3,4dicarboxyphenoxy) phenyl]propane dianhydride (BPADA) with 4,4'-oxydianiline (ODA).

tion. The dissolved polymer acts like surfactant for the synthesized nanoparticles which indicate good dispersion in the solution. Dissolved polymer molecules create tiny reaction chambers for nanoparticle synthesis. After solvent casting and drying films, the synthesized particles are randomly distributed, and stablized (frozen in location) in a polymer matrix. $\mathrm{TiO}_{2}$ particle polyphenylsulphone in-situ nanocomposite with good nanoparticle dispersion and size distribution is shown in Figure 8. The cast film was 25 micron thick.

It was noted that interface fraction becomes increasingly important when the particles are much smaller. According to the computation made by Raetzke et al. [20], the particle interface becomes significantly high when the particle sizes are below $10 \mathrm{~nm}$, see also Tuncer [18]. Figure 9 is the re-plot of the relationship between particle interface and particle size under various filler contents. For particles of $10 \mathrm{~nm}$, the composite with $5 \mathrm{vol} \%$ of particles contains about $40 \mathrm{vol} \%$ interfaces. The $5 \mathrm{~nm}$

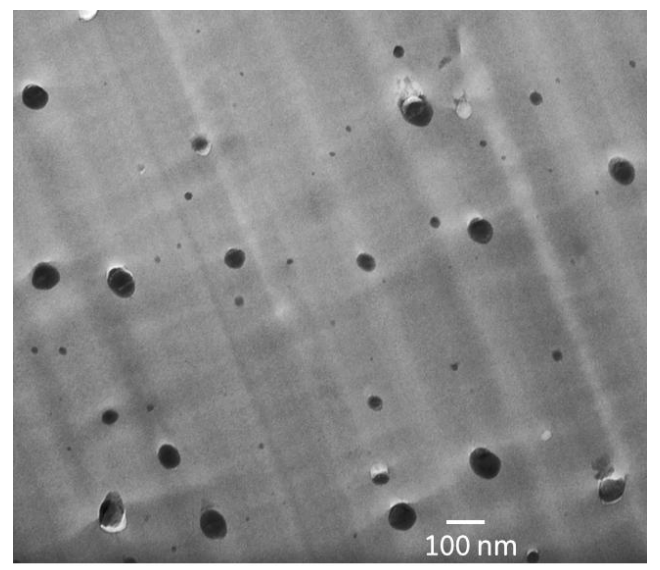

Figure 8. TEM image of $5 \mathrm{wt} \% \mathrm{TiO}_{2}$ nanoparticles dispersed in a PPSU film using a self-assembled in-situ particle precipitation process. Nanoparticles nucleated at rondom locations in the matrix that yield well-dispersion of particles without any agglemoration.

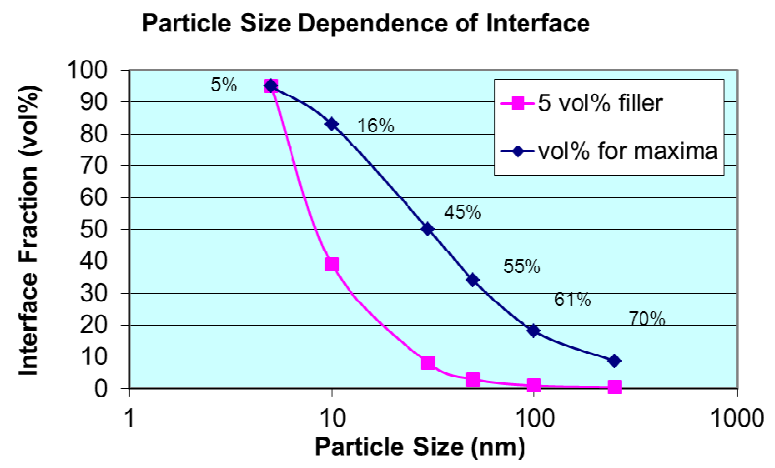

Figure 9. Particle size dependence of interface fraction of nanoparticles in a polymer matrix. The blue curve represents the volume percentage of particle required to reach the maximum interface fraction for various particle sizes.

particles can result in 95 vol\% interface of the composites. For $10 \mathrm{~nm}$ particles, $15 \mathrm{vol} \%$ particles could result in more than $85 \%$ of interface. Tuncer et al. also reported very high particle density and shorter particle-particle distance at lower volume percent of particles [18]. As a result, even very small amount of particles would result in percolation where particle interconnection occurs, which would be very unlikely due to required statistical outcomes for the given size and thickness.

Experimental evidence of high interface and particle interconnection was obtained from the cyanothyl cellulose film as shown in Figure 10. When 20 vol\% of $8 \mathrm{~nm}$ $\mathrm{BaTiO}_{3}$ particles was added, it is clear that the particles agglomerate and form large clusters, which would be the reason for the observed high dielectric permittivity-non-spheroidal shapes in the field direction.. Particles are hard to be observed in the bulk; they introduced high interface regions. Previous investigations have shown that $\mathrm{BaTiO}_{3}$ particles smaller than $15 \mathrm{~nm}$ can lose most 


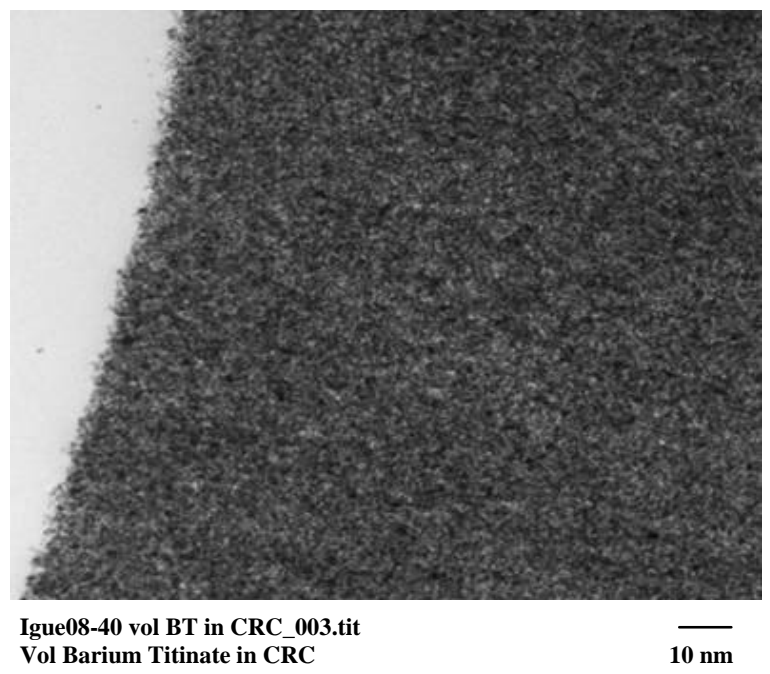

Figure 10. TEM images of highly concentrated $\mathrm{BaTiO}_{3}$ nanoparticles $(20 \mathrm{vol} \%)$ in cyanothyl cellulose. The particles are clearly visible on the edge, otherwise it is hard to differentiate the particles in the bulk.

of their ferroelectricity, and therefore their dielectric constant is drastically lowered [21]. Further improvements in synthesis, purity and size control of nanoparticles are required to enable a better performance of the nanodielectric composites.

\section{Dielectric Properties of Nanocomposites}

We should concentrate how we can better utilize nanofillers and created high internal surfaces. When the particles are not interconnected, the contribution of the individual particles to the composite permittivity is limited; this is true for spherical particles. When permittivity ratio between the inclusion particles and the matrix is large, the effective permittivity of the composite would be close to the matrix permittivity below the percolation threshold [22]. At percolation threshold and above the particles would have high probability to form interconnections (clusters) union of particles act as non-spheroidal (if they were spheroidal initially) inclusions. If the union of the particles is in the direction of the applied electrical field, they would yield high polarization/depolarization values due to these effective particle shapes. In addition depending on the frequency of interest the interfacial polarization would play an additional role in the polarization, thus the effective permittivity of the composite. In 3-dimensions the percolation threshold for spherical particles is about $1 / 3$; however, remember that the shape of inclusions will change the threshold value, which is the case when particle clusters are formed.

If we reconsider the interfacial contribution once again, embedding 5 vol\% of $20 \mathrm{~nm}$ particles would only yield about $10 \%$ interface, if the interface has some contribution to the permittivity other than the known interfacial polarization (or Maxwell-Wagner-Sillars polarization).

GRC found that adding $5 \%$ of colloidal $\mathrm{SiO}_{2}$ particles in a PEI film only increased the permittivity to 4 (i.e., $25 \%$ higher); however due to solvent casting procedure we anticipate that there either were ionic species or residual solvent that result in a higher permittivity for the composite than the constituents-PEI and $\mathrm{SiO}_{2}$ have relative permittivities 3.2 and 3.9, respectively. Similarly, adding 5 vol\% of $\mathrm{BaTiO}_{3}$ nanofillers in the cyanoethyl cellulose (CR), as described in Figure 9, increased the permittivity only by $25 \%$ in spite of the high permittivity of $\mathrm{BaTiO}_{3}$. When the particle loading exceeds the percolation level, the permittivity was significantly increased by more than 5 times as shown in Figure 11 [23]. Therefore, the direct contribution of the interconnected particles is most important to the increase of composite permittivity. When the nanoparticles were very small, we have found that the interconnection between particles did not cause mechanical brittleness; polymer can cover the particles depending on the size of the particles and the gyration radius of polymer chains.

Interfacial contribution, particle surface chemistry and contamination due to ionic species become important in nanocomposites that alter the electric properties of a composite, effective permittivity and conductivity, and also the dielectric breakdown strength. The interface contribution should be studied not only on its nature but also the way it affect the composite. Todd et al. investigated the effect of coupling agents on the interphase dielectric constant using experimental data and molecular dipole polarization calculations [24]. They have shown that the dielectric characteristics of polymer-ceramic interphase regions affect the overall dielectric response of the composite system. The interphase region depends largely on the chemical bonding at the filler interface that is capable of forming covalent or hydrogen bonds to the surface of the filler. The material in the interface has a different dielectric characteristics; it is an interphase region of a polymer-ceramic composite.

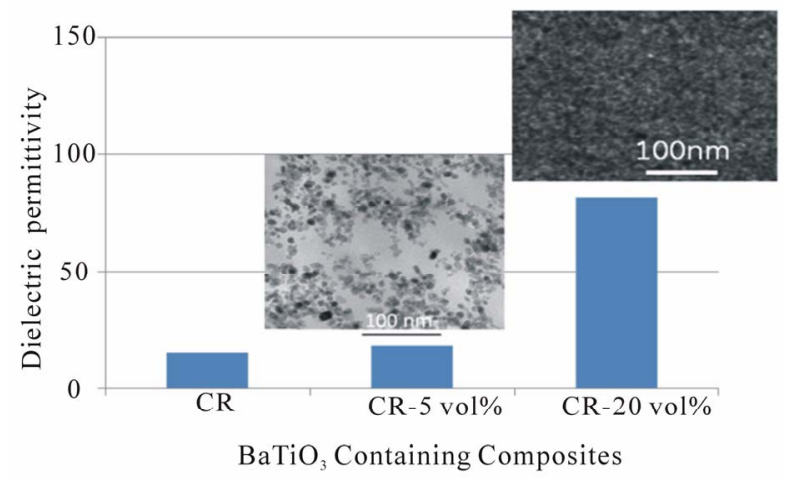

Figure 11. $\mathrm{BaTiO}_{3}$ nanoparticle loading effect on the permittivity of the composites. 
The dynamic response of the particle interfaces was studied by Tan et al. using a dielectric spectroscopy technique capable of working on a wide frequency and temperature range [25]. For the selected base polymer (PEI) containing no particles, and thus, no interfaces (interfacial polarization), the dielectric loss spectra exhibit low loss tangents of the polymer (Figure 12). The permittivity of the PEI is not shown since in the considered frequency and temperature ranges it has small dispersion also reflected in the loss tangent plots, which are lower than $1 \%$. The dielectric properties of the PEI produced by extrusion and solvent (spin) casting are shown to indicate the differences in the material due to processing. However as shown in Figure 12, there are only slight differences at low temperatures (below $50^{\circ} \mathrm{C}$ ) and high temperatures above $200^{\circ} \mathrm{C}$. The PEI polymer is an excellent material for high temperature capacitor applications because of low loss tangent over broad temperature and frequencies.

Influence of particles and particle size on the dielectric loss tangent spectra were investigated as shown in Figure 13. The dielectric behavior of the polymer was altered with addition of different size of alumina particles. The particle concentration was 5 vol\% in the investigations. Besides the sample with $45 \mathrm{~nm}$ particles, the loss

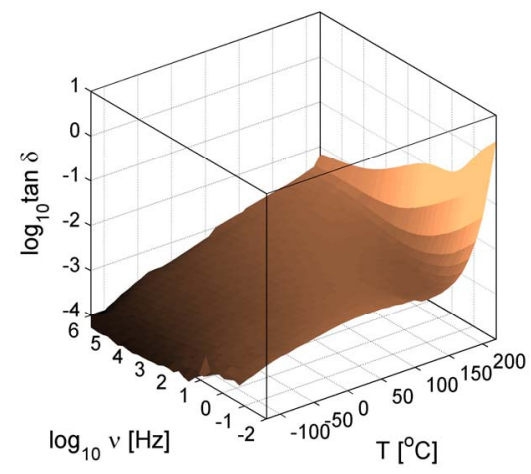

(a)

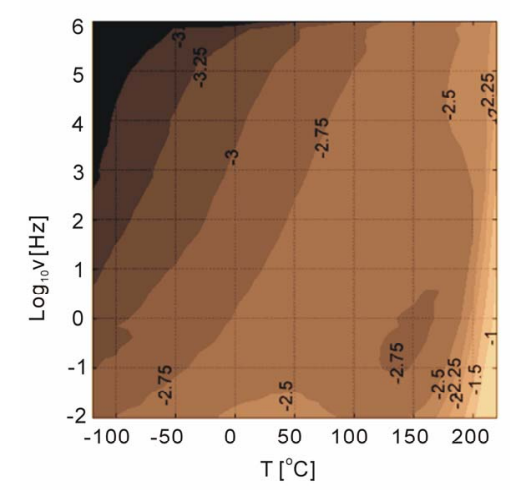

(c) tangent behavior at high temperatures and low frequencies were similar to unfilled PEI. We anticipate that the nanocomposite sample with $45 \mathrm{~nm}$ alumina had defects that generated high losses at high temperatures and low frequencies, which could be a results of charge conduction introduced with the defects. We discuss this later in the text. The smallest particles that were considered in the investigations were $11 \mathrm{~nm}$ and the composite had a loss peak at high frequencies probably due to the large surface area introduced by the alumina nanoparticles, which could be related to the interfacial polarization. The composites with large particles, $150 \mathrm{~nm}$ and 1 micron, illustrate that the changes in the dielectric loss tangent behaivor were higher than the unfilled PEI. The loss tangents between $150 \mathrm{~nm}$ and 1 um particle samples were similar with 1um particles generating slightly more loss tangents at high tempatures and low frequencies.

Size of nanoparticles has known to alter the dielectric behavior of sintered ceramics [26], such that small nanoparticles yield low dielectric permittivity sintered materials. This observation brings the question that as particle size is decreased what would be the permittivity of individual nanaparticles. Or in other words, would we observe similar dielectric behavior with similar size but

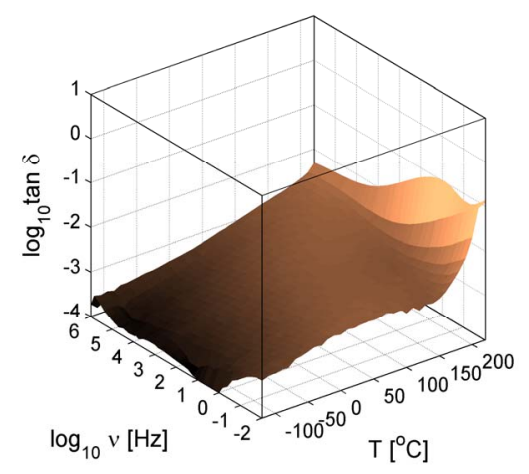

(b)

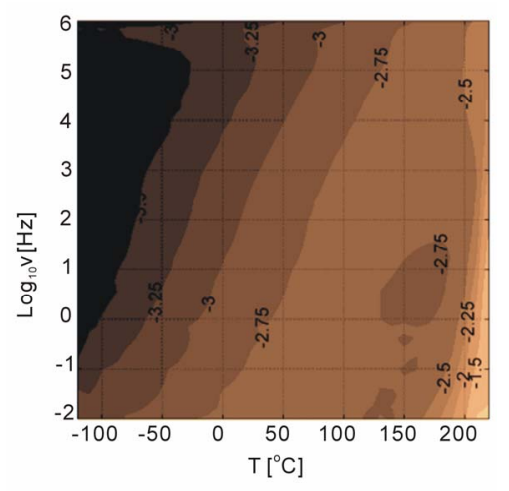

(d)

Figure 12. Dielectric loss tangent of PEI as a function of temperature and frequency. The three dimensional surface plots in (a) and (b) are shown in contour plots in (c) and (d) to help the readers to compare the loss behavior of unfilled material with those of filled material. (a) and (c) are for extruded PEI and (b) and (d) are for in-house solvent casted PEI. 
different nanoparticles? Similarly one can ask what would be the electrical conductivity of the nanoparticles; size dependent conductivity. There is no data in the literature on this topic to our knowledge.

To study the type of particle and its influence on the dielectric loss tangent behavior of alumina and antimony oxide $\mathrm{Sb}_{2} \mathrm{O}_{3}$ particles were investigated for $5 \mathrm{vol} \%$ nanoparticle filled systems. The loss tangent is shown in Figure 14 where the antimony oxide has higher losses than alumina. Although the relative dielectric permittivities of the two fillers are slightly similar, 10 for alumina and 12.4 for antimony trioxide [27] yielding similar permittivity ratios between the filler and the polymer-the electrical conductivities of the two fillers are different. The ratio of conductivies between PEI and alumina and PEI and antimony trioxide can be considered as 1 and 10 5 , respectively, considering that the polymer and alumina have around $10-12 \mathrm{~S} / \mathrm{m}$, however antimony trioxide has $10-7 \mathrm{~S} / \mathrm{m}$ [28]. This would generate a high loss due to the mismatch in the dielectric permittivities. While it is clear from Figure 14 that the differences in the conductivities did not result in a significant loss tangent change in the dielectric response. Both $11 \mathrm{~nm}$ size fillers have a similar response considering that the behavior at high frequencies are similar. This observation indicates

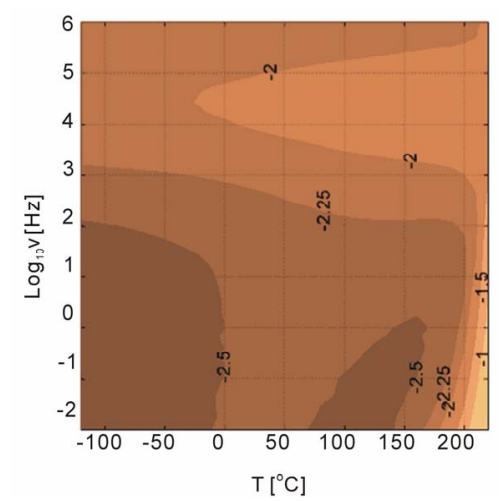

(a)

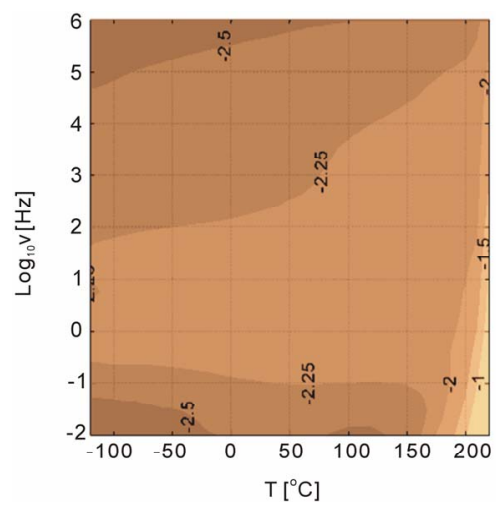

(c) that perhaps the size of the particles are more important than the chemical nature of the particles.

As mentioned previously the purity of particles are important. For example, the particle surface contamination was determined using an analytical technique (XPS) [24], and concluded that the raw particles need to be cleaned up before blending into polymers. Presence of impurities on particle surfaces becomes detrimental dielectric performance under high electric fields (low dielectric breakdown strength). The data for the composite sample with $45 \mathrm{~nm}$ alumina shows the signs of this effect, see Figure 13. A cyanothyl cellulose polymer containing.

$\mathrm{BaTiO}_{3}$ or $\mathrm{PbZrO}_{3}$ particles was found to exhibit 50\% higher breakdown strength when the particles were pretreated to remove surface contaminants [29]. Therefore, properly preparing the nanoparticles is critical for the augmentation of the dielectric properties of nanocomposites. Depending on the density of the conteminants, their influence can be detected in loss tangent via dielectric spectroscopy.

\section{Discussion}

To achieve improvements in the breakdown strength of nanocomposite have been mostly difficult over the years.

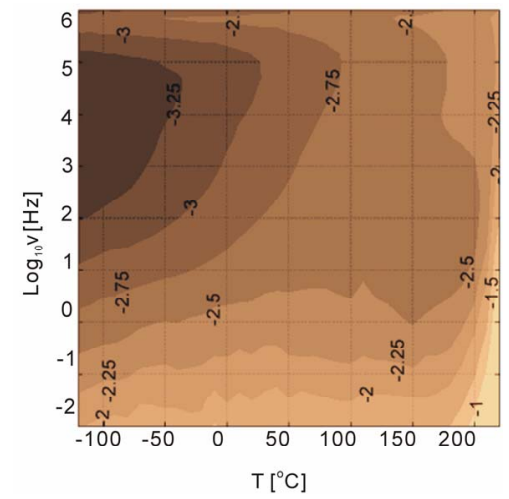

(b)

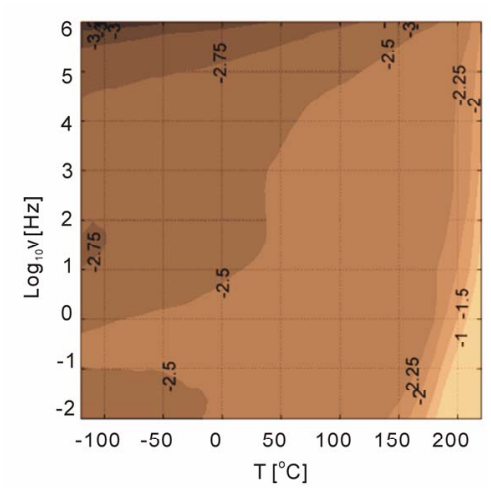

(d)

Figure 13. Dielectric loss tangent of PEI filled with alumina particles as a function of temperature and frequency. The size of particles is altered in the experiments, (a) $11 \mathrm{~nm}$, (b) $45 \mathrm{~nm}$ (c) $150 \mathrm{~nm}$ and (d) $1 \mathrm{um}$. The materials are casted in-house. 


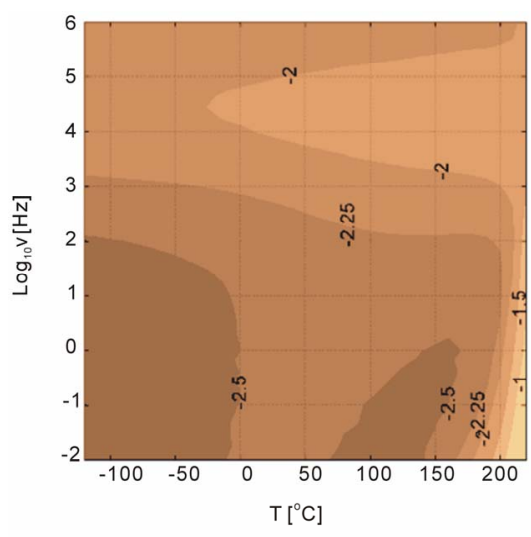

(a)

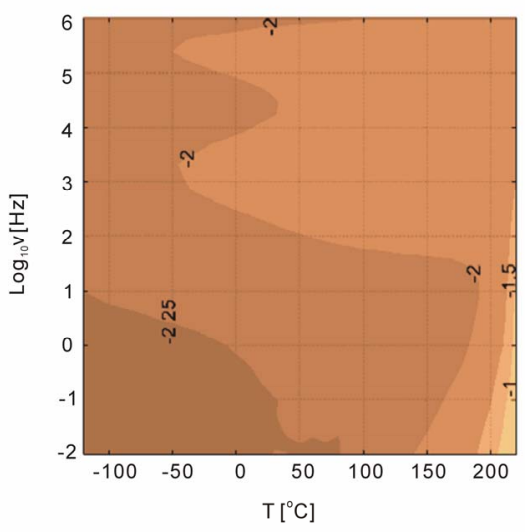

(b)

Figure 14. Dielectric loss tangent for (a) $11 \mathrm{~nm}$ alumina and $11 \mathrm{~nm}$ antimony trioxide filled PEI as functions of temperature and frequency.

In most cases, the breakdown strength equivalent to that of the base polymers were attained when the particles are well-dispersed as shown in micrographs as in Figures 6-8 and 10 either by wet particle or in-situ particle precipitation methods. It has become a commonalty that higher loading would result in degradation in the breakdown strenght of the composites, due to increase number of particle aggregates. Therefore, leveraging low particle concentrations and high interface contribution associated with small nanoparticles would be a preferred method for equivalent or higher breakdown strength nanocomposites compared to unfilled matrix. As discussed previously, a good example of well-dispersed nanoparticles can be achieved byself-assembly of metal-oxides, see for example $\mathrm{TiO}_{2}$ nanocompositesby Tuncer et al. who reported higher nanocompositebreakdown strength values than that of neat polymers $[12,13]$. Similar work with an epoxy nanocomposite reporteda breakdown strength increase by $20 \%$ $[12,30]$.

Recently, breakdown strength of $>700 \mathrm{kV} / \mathrm{mm}$ was achieved in thermoplastic films. These results demonstrated a promising method for reaching higher energy den- sity materials than the conventional thermoplastics. Better engineering of nanocomposites and processing methods should be developed to achieve both higher per- mittivity and breakdown strength.

\section{Conclusion}

A brief review of polymeric nanodielectric composites work performed at GE GRC in Niskayuna NY was presented. The filler functionalization, distribution and dis persionhave clearly become major focus of interests in the nanodielectric research. It was found that the status of nanofiller precursors is critical to create well-defined nanostructures and desired or required dielectric and electrical properties. Successes in nanoparticle dispersion in nanocomposites require a solution chemistry and nanoparticle precursors. Examples for successful nanocomposite need wet particles, clean particle surface, suitable mixing methods such as in-situ and self-assembly. The on-going investigations at our Labsshow that a high dielectric permittivity and/or breakdown strength in nanodielectric composites could be achieved, if proper nanofillers and processing methods are utilized. For in-situ polymerization or precipitation processes, well-dispersed particles and precursors in a solution are desired; however, the discussed processes, development of their scaleup and production of nanoparticles and polymeric films still need significant efforts. For the presented self-assembled nanocomposites process, parameters and conditions are still in progress.

\section{Acknowledgements}

Support from DARPA DSO under a Contract FA945108-C-0166 is greatly acknowledged. Discussion on Barium Titanate nanoparticles with Professor Steven O’Biren and Dr. Limin Huang at Columbia University are greatly appreciated.

\section{REFERENCES}

[1] A. S. Arico, P. Bruce, B. Scrosati, J.-M. Tarascon and W. Van Schalkwijk, "Nanostructured Materials for Advanced Energy Conversion and Storage Devices," Nature Materials, Vol. 4, No. 5, 2005 pp. 366-377. doi:10.1038/nmat1368

[2] Y. Cao, P. Irwin and K. Younsi, "The Future of Nanodielectrics in the Electrical Power Industry,” IEEE Transactions on Dielectrics and Electrical Insulation, Vol. 11 No. 5, 2004, pp. 797-807.

[3] N. Guo, S. A. DiBenedetto, D. K. Kwon, L. Wang, M. T. Russell, M. T. Lanagan, A. Facchetti and T. J. Marks, "Supported Metallocene Catalysis for in Situ Synthesis of High Energy Density Metal Oxide Nanocomposites," Journal of the American Chemical Society, Vol. 129, No. 4, 2007, pp. 766-767. doi:10.1021/ja066965l 
[4] K. J. Nelson, "Dielectric Nanocomposite Polymers,” Springer, New York, 2009.

[5] E. Tuncer, G. Polizos, I. Sauers, D. R. James, A. R. Ellis and K. L. More, "Epoxy Nanodielectrics Fabricated with In-Situ and Ex-Situ Techniques,” Journal of Experimental Nanoscience, Vol. 7, No. 3, 2011, pp. 274-281. http://www.tandfonline.com/doi/abs/10.1080/17458080.2 010.520137

[6] Y. Bai, Z.-Y. Cheng, V. Bharti, H. S. Xu and Q. M. Zhang, "High-Dielectric-Constant Ceramic-Powder Polymer Composites," Applied Physics Letters, Vol. 76, No. 25, 2000, p. 3804.

[7] E. A. Cherney, "Silicone Rubber Dielectrics Modified by Inorganic Fillers for Outdoor High Voltage Insulation Applications," Annual Report Conference on Electrical Insulation and Dielectric Phenomena, Nashville, 16-19 October 2005, pp. 1, 9.

[8] D. Q. Tan, Y. Cao and P. Irwin, "Nanostructured Dielectric Materials," International Conference on Solid Dielectrics, Winchester, 8-13 July 2007, pp. 411-414.

[9] P. Kim, S. C. Jones, P. J. Hotchkiss, J. N. Haddock, B. Kippelen, S. R. Marder and J. W. Perry, "Phosphonic Acid-Modified Barium Titanate Polymer Nanocomposites with High Permittivity and Dielectric Strength,” Advanced Materials, Vol. 19, No. 7, 2007, pp. 1001-1005. doi:10.1002/adma.200602422

[10] E. Tuncer, "Structure/Property Relationship in Dielectric Mixtures: Application of the Spectral Density Theory," Journal of Physics D: Applied Physics, Vol. 38, No. 2, 2005, pp. 223-234. doi:10.1088/0022-3727/38/2/006

[11] S. Takahashi and D. R. Paul, "Gas Permeation in Poly (Ether Imide) Nanocomposite Membranes Based on Surface-Treated Silica. Part 2: With Chemical Coupling to Matrix,” Polymer, Vol. 47, No. 21, 2006, pp. 7535-7547.

[12] E. Tuncer, I. Sauers, D. R. James, A. R. Ellis, M. P. Paranthaman, A. Goyal and K. L. More, "Enhancement of Dielectric Strength in Nanocomposites," Nanotechnology, Vol. 18, No. 32, 2007, Article ID: 325704.

[13] E. Tuncer, I. Sauers, D. R. James, A. R. Ellis, M. Pace, K. L. More, S. Sathyamurthy, J. Woodward and A. J. Rondinone, "Nanodielectrics for Cryogenic Applications," IEEE Transactions on Applied Superconductivity, Vol. 19, No. 3, 2009, pp. 2354-2358. doi:10.1109/TASC.2009.2018198

[14] E. Tuncer, A. J. Rondinone, J. Woodward, I. Sauers, D. R. James and A. R. Ellis, "Cobalt Iron-Oxide Nanoparticle Modified Poly(Methyl Methacrylate) Nanodielectrics: Dielectric and Electrical Insulation Properties,” Applied Physics A, Vol. 94, No. 4, 2009, pp. 843-852. doi:10.1007/s00339-008-4881-8

[15] R. A. Vaia and E. P. Giannelis, "Polymer Nanocomposites: Status and Opportunities,” MRS Bulletin, Vol. 26, No. 5, 2001, pp. 394-401. doi:10.1557/mrs2001.93

[16] S. H. Zhang, Y. Cao, D. Tan and P. Irwin, "Nanodielectric Ultem Films,” GE Internal Report, Publishing House GE Global Research Center, Niskayuna, 2006.

[17] Y. Cao, Q. Chen, D. Q. Tan and P. C. Irwin, "Nanostructured Dielectric Materials,” International Conference on
Solid Dielectrics, Winchester, 8-13 July 2007, p. 163.

[18] E. Tuncer, A. J. Rondinone, J. Woodward, I. Sauers, D. R. James and A. R. Ellis, "Cobalt Iron-Oxide Nanoparticle Modified Poly(Methyl Methacrylate) Nanodielectrics," Applied Physics A, Vol. 94, No. 4, 2009, pp. 843-852. doi:10.1007/s00339-008-4881-8

[19] G. Polizos, E. Tuncer, I. Sauers and K. L. More, "Properties of a Nanodielectric Cryogenic Resin,” Applied Physics Letters, Vol. 96, No. 15, 2010, Article ID: 152903. doi:10.1063/1.3394011

[20] S. Raetzke and J. Kindersberger, "The Effect of Interphase Structures in Nanodielectrics,” IEEJ Transactions on Fundamentals and Materials, Vol. 126, No. 11, 2006, pp. 1044-1049. doi:10.1541/ieejfms.126.1044

[21] L. M. Huang, J. Zhang, I. Kymissis and S. O’Biren, "High $\mathrm{K}$ Capacitors and OFET Gate Dielectrics from Self-Assembled $\mathrm{BaTiO}_{3}$ and $(\mathrm{Ba}, \mathrm{Sr}) \mathrm{TiO}_{3}$ Nanocrystals in the Superparaelectric Limit,” Advanced Functional Materials, Vol. 20, No. 4, 2010, pp. 554-560. doi:10.1002/adfm.200901258

[22] A. H. Sihvola, "Electromagnetic Mixing Formulas and Applications Ari Sihvola,” IEE Publication Series, IEE, London, 2000.

[23] D. Q. Tan, Q. Chen, Y. Cao, P. Irwin and S. Heidger, "Polymer Based Nanodielectric Composites for Capacitors," Presentation at American Ceramic Society Electronic Materials and Applications, Orlando, Unpublished.

[24] M. G. Todd and F. G. Shi, "Characterizing the Interphase Dielectric Constant of Polymer Composite Materials: Effect of Chemical Coupling Agents,” Journal of Applied Physics, Vol. 94, No. 7, 2003, p. 4551.

[25] D. Q. Tan, Y. Cao, P. Irwin, K. Shuman and C. McTigue, "Interfacial Study of Nanoparticle Filled Polyetherimide," Presentation at International Conference on Materials (IUMRS-ICM), July 2008.

[26] T. Tsurumi, T. Hoshina, H. Takeda, Y. Mizuno and H. Chazono, "Size Effect of Barium Titanate and Computer-Aided Design of Multilayered Ceramic Capacitors," IEEE Transactions on Ultrasonics, Ferroelectrics, and Frequency Control, Vol. 56, No. 8, 2009, pp. 1513-1522. doi:10.1109/TUFFC.2009.1214

[27] M. M. Abou Sekkina, "Effects of Temperature and Frequency Changes on the Dielectric Properties of Modified Chalcogenides,” Thermochimica Acta, Vol. 120, 1987, pp. 231-239.

[28] N. Tigau, V. Ciupina, G. Prodan, G. I. Rusu, C. Gheorghies and E. Vasile, "The Influence of Heat Treatment on the Electrical Conductivity of Antimony Trioxide Thin Films," Journal of Optoelectronics and Advanced Materials, Vol. 5, No. 4, 2003, pp. 907-912.

[29] D. Q. Tan, “Intergrated High Energy Density Capacitors,” GE Internal Report, GE Global Research Center, Niskayuna, 2010.

[30] E. Tuncer, I. Sauers, D. R. James, A. R. Ellis, M. P. Paranthaman, T. Aytug, S. Sathyamurthy, K. L. More, J. $\mathrm{Li}$ and A. Goyal, "Electrical Properties of Epoxy Resin Based Nanocomposites,” Nanotechnology, Vol. 18, No. 2, 2007, Article ID: 025703. doi:10.1088/0957-4484/18/2/025703 